\title{
ESCALA DE ACTITUDES HACIA LA TECNOLOGÍA EN LA LABOR DOCENTE APLICADA A PROFESORES DE PRIMARIA DE INFORMÁTICA EDUCATIVA EN COSTA RICA ANÁLISIS DE VALIDEZ Y CONFIABILIDAD
}

\author{
Rodrigo Vargas Ruiz*
}

En este documento se presenta el resultado del análisis de validez y confiabilidad de la Escala de Actitudes hacia la Tecnología en la Labor Docente, aplicada a 319 tutores y maestros de informática educativa de educación primaria pública en Costa Rica, cuyos centros educativos están adscritos al Programa Nacional de Informática Educativa para I y II Ciclos del Ministerio de Educación Pública y de la Fundación Omar Dengo. La escala inicial estuvo conformada por 115 ítemes divididos en once categorías. Los análisis realizados permitieron un reordenamiento de las categorías de la escala y desechar varios ítemes cuyas cargas factoriales eran inferiores a 0.4 y correlaciones inferiores a 0.3. En el análisis de confiabilidad se obtuvo un Alfa de Cronbach de 0.8985 para concluir que la escala final tiene una muy buena consistencia interna. Finalmente, con los resultados obtenidos se construyó una nueva escala con 41 ítemes agrupados en siete categorías y estas en dos dimensiones.
In this document, the results of the validit and reliability analy'sis of the constructed "Attitudes toward Technology in Educational School Work" scale, are presented. Data were collected from 319 educative computer science teachers of Costa Rican public primary schools, which take part in the National Program of Educational Informatics for Primary Education, jointly implemented by the Costa Rican Ministry of Public Education and the Omar Dengo Foundation. The scale was primarily composed of 115 items, which were divided in eleven categories. The analysis allowed a reordering scale categories and remove several items with factor loadings lower 0.4 and correlations lower 0.3. Reliability analy'sis gave as result a Cronbach coefficient of 0.8985 , leading to the conclusion that the scale is reliable. Finally, taking into account the obtained results, a new scale was constructed with 41 items, gathered in seven categories, and these divided in two dimensions.

* Estadístico y Magíster Scientiae en Antropología de la Universidad de Costa Rica. Desde 1994 se ha desempeñado como investigador y ha llevado a cabo diversos estudios relacionados con la salud, las sexualidades y la cultura, tanto en Costa Rica como en América Central. Fue Director de Investigación del Instituto Latinoamericano de Prevención y Educación en Salud (ILPES), investigador y Director a.i. del Departamento de Investigación de la Fundación Omar Dengo, y actualmente se desempeña como investigador del Instituto de Estudios Sociales en Población (IDESPO) de la Universidad Nacional. Su publicación más reciente (2003) es el libro "Pétalos y espinas. Hombres gay, relaciones de pareja y violencia". 
Este trabajo forma parte del proyecto de investigación "La educación y las TIC: desarrollo de una metodología para evaluar impacto social y condiciones de equidad", patrocinado por el Centro Internacional de Investigaciones para el Desarrollo (CIID/IDRC) y desarrollado por el Departamento de Investigación de la Fundación Omar Dengo. El proyecto tiene como objetivo general desarrollar una propuesta metodológica que permita evaluar el impacto social de proyectos educativos fundamentados en la utilización de las Tecnologías de la Información y la Comunicación (TIC), desde la perspectiva de su contribución a la generación de condiciones de equidad.

Para el cumplimiento de este objetivo se elaboró, en primer lugar, un marco organizativo para la evaluación del impacto social con perspectiva de equidad', organizado por ámbitos y aspectos que permitieran clasificar un importante listado de indicadores, señalados en la literatura en este campo, y finalmente proceder con la construcción de estrategias que colaboraran con esa evaluación.

Este documento presenta el análisis de validez y confiabilidad para la depuración de la Escala de actitudes ${ }^{2}$ hacia la Tecnología en la Labor Docente (EATLD) creada para ser aplicada a maestros (as) y tutores (as) de informática educativa (IE) de la primaria pública en Costa Rica.

La población a la cual se aplicó la escala, después de una prueba de campo para su depuración inicial, fue de 319 maestros (as) y tutores (as) de IE de primaria, cuyos centros educativos están inscritos en el PRONIE I y II Ciclos MEPFOD y que asistieron al IX Congreso Nacional Infantil de Informática Educativa el 28 y 29 de marzo del 2003 en Santa Cruz, Guanacaste.

La EATLD que se utilizó fue construida a partir de diferentes consultas bibliográficas realizadas y por las experiencias previas del Departamento de Investigación, tales como que la tecnología tiene una incidencia en la motivación de maestros y tutores en IE para asistir a la institución o como qué programas educativos que se fundamentan en el uso de las TIC tienen un impacto en la visión de futuro.

La definición de actitud que se ha asumido es la de Thurstone (1978) es: "La suma total de inclinaciones, sentimientos, prejuicios o distorsiones, nociones preconcebidas, ideas, temores, amenazas y convicciones de un individuo acerca de cualquier asunto específico" (Thurstone, 1978: 158, mencionado en

1 Para una profundización en el marco desarrollado refiérase a Rodríguez. J. (2002). Marco evaluativo de impacto social con perspectiva de equidad para programas educativos mediados por TIC. Departamento de Investigación, Fundación Omar Dengo.

2 En el documento Actitudes de docentes y estudiantes hacia la tecnología en la educación y el aprendizaje: una evaluación comparativa entre beneficiados y no beneficiados del Programa Nacional de Informática Educativa para I y II Ciclos MEP-FOD, de Castro, R. (2003), se expone la conceptuación alrededor de las actitudes y su medición, específicamente en relación con el aprendizaje de la tecnología. 
Castro, 2003). Las actitudes atraviesan la actividad humana y tienden a orientar los procesos perceptuales, cognitivos y emotivos implicados en el aprendizaje. De ahí la importancia de su estudio como parte de los factores que median en el impacto obtenido por programas educativos apoyados en la TIC.

Con estas consideraciones se realizó una revisión bibliográfica en la que se identificó una serie de aspectos importantes para evaluar las actitudes hacia la tecnología en la educación, tales como los planteados por Lignan (1999, 2002); Mitra (2002), Franton, Green y Hoffman (2002) y Myint Swe Khine (2001). A estos aspectos se agregaron los identificados en estudios realizados por el Departamento de Investigación de la Fundación Omar Dengo, obteniéndose el siguiente listado:

1. Valoración de la tecnología

2. Motivación para asistir a la institución a partir de la introducción de las TIC

3. Satisfacción con el programa o actividad

4. Autopercepción de la capacidad para enseñar

5. Autopercepción profesional

6. Visión de futuro

7. Planteamiento de metas de superación continuas

8. Actitud para participar en grupos

9. Sentido de control que se tiene sobre la tecnología

10. Valoración del aprendizaje de la tecnología

11. Valoración de la acción pedagógica

12. Motivación para aprender

13. Autorregulación de la relación con la tecnología

14. Autonomía en la relación con la tecnología

15. Aprendizaje de nuevas habilidades relacionadas con la tecnología

16. Percepción sobre las ventajas o inconvenientes de la propuesta del Programa 17. Gestión de medios externos

A partir de este conjunto se construyeron las categorías de la EATLD que se presentạán más adelante con sus respectivos ítemes.

\section{Validez y confiabilidad}

Antes de iniciar la presentación de las pruebas estadísticas que se utilizaron para depurar y construir la EATLD final, se vuelve necesario exponer qué se entiende por validez y confiabilidad. 
La validez de una escala se refiere a lo que mide y cómo lo mide (¿el instrumento mide lo que se pretende medir?). Las escalas se diseñan para propósitos concretos y, desde este punto de vista, no existe la escala perfecta para cuantificar cualquier aspecto. Por lo tanto, no se puede hablar de validez de una escala en términos generales, mencionando que es alta o baja. sino que se determinará respecto de los objetivos específicos para los que fue diseñada.

Por otro lado, la confiabilidad de una escala indica la consistencia de los resultados de los mismos individuos cuando son evaluados en diferentes ocasiones. Al ser la fiabilidad una medida del grado de consistencia o concordancia, puede expresarse siempre mediante algún coeficiente de correlación. Está relacionada con la estabilidad o constancia, con la coherencia o consistencia interna y la precisión de las medidas que se obtienen con el instrumante. Es importante recalcar que cualquier coeficiente de fiabilidad depende tanto de la escala como de la variabilidad de la muestra.

En cualquier tipo de investigación, la validez y la fiabilidad de los instrumentos de recolección de información son fundamentales para la calidad de los datos obtenidos. Usar una escala como instrumento de recolección de datos necesariamente implica controlar la fiabilidad y la validez. Estas dos condiciones son cruciales porque si las herramientas de recolección usadas fueron defectuosas hay que olvidarse del éxito de la investigación, dado que el tratamiento estadístico no conseguirá el milagro de transformar datos de mala calidad en buenos resultados.

\section{Las pruebas estadísticas aplicadas}

El análisis que se ha aplicado para la depuración final de la EATLD tiene su fundamentación en tres pruebas estadísticas.

La primera de ellas es el análisis de factores (o factorial) que consiste en identificar variables subyacentes, o factores, que expliquen la configuración de las correlaciones dentro del conjunto de variables observadas. El análisis de factores se suele utilizar en la reducción o simplificación de datos para identificar un pequeño número de factores que explique la mayoría de la varianza observada en un número mayor de variables manifiestas.

En otras palabras, y para el caso que compete a la EATLD, este método estadístico permite precisar el número y naturaleza de los ítemes que forman una determinada categoría. Debe recordarse que las categorías fueron construidas previamente para desglosar el objeto de estudio. Con este análisis se generan "variables artificiales", conocidas también como variables latentes o dimensiones, (categorías compuestas por ítemes) que representan los factores. Los factores se obtienen de los ítemes originales y se interpretan de acuerdo 
con el significado de las categorías. En resumen, “... es una técnica para explicar un fenómeno complejo en función de un determinado grupo de variables." (Hernández, Fernández y Baptista. 2000: 414).

Debido a que, como se mencionó, las categorías de la escala (con sus respectivos ítemes) fueron construidas previamente desde la teoría presentada por diversos autores y desde las hipótesis de los investigadores, el análisis de factores permite validar esa construcción.

La validez del constructo se determina investigando las cualidades sicológicas, rasgos o factores evaluados por un test. La validez factorial es un ejemplo de ello. Los que conciben los tests para medir capacidades y adaptaciones han demostrado que los rasgos estudiados pueden reducirse a elementos estadísticos llamados factores. (...) Un test correlacionado significativamente con tales factores tiene validez factorial, un tipo de validez de construcción. Esta última puede ser tanto lógica como estadística. (Downie, 1973: 266).

El análisis de factores proporciona una medida de la homogeneidad no solo de la totalidad de ítemes que conforman la escala sino del grupo de ítemes que se registran dentro de una categoría. Además, a través de la medición de esa homogeneidad se puede determinar si los ítemes "explican" el comportamiento de esa categoría y si alguno debería estar adscrito a otra categoría y no a la que pertenece originalmente.

La prueba cumple su cometido cuando se analizan las cargas factoriales de los ítemes de cada una de las categorías. Se ha determinado con la teoría y a través de la práctica, que cargas factoriales inferiores a 0.4 (pueden variar entre -1 y +1 ) generan una muy baja correlación ítem-total, de ahí que deban desecharse para aumentar la confiabilidad de la escala.

La segunda prueba está relacionada con el análisis de la correlación de todos los ítemes de la escala en cada una de las categorías (todos comparados con todos, en pares). Para realizarla se utiliza el Coeficiente de Correlación de Pearson (correlación ítem-total), que permite obtener la relación de dos variables medidas por intervalos o de razón.

Este coeficiente indica la magnitud y la dirección de la relación entre los ítemes y proporciona una medida de la fuerza con que están relacionados. Su valor puede oscilar entre -1 y +1 . El valor -1 significa que hay una correlación negativa perfecta (conforme aumenta el puntaje de un ítem, el otro disminuye); 0 quiere decir que no existe correlación alguna y +1 indica una correlación positiva perfecta entre los ítemes (conforme uno aumenta su puntaje, el otro también). 
Este documento presenta el análisis de validez y confiabilidad para la depuración de la Escala de actitudes hacia la Tecnología en la Labor Docente (EATLD) creada para ser aplicada a maestros (as) y tutores (as) de informática educativa (IE) de la primaria pública en Costa Rica... La EATLD que se utilizó fue construida a partir de diferentes consultas bibliográficas realizadas y por las experiencias previas del Departamento de Investigación, tales como que la tecnología tiene una incidencia en la motivación de maestros y tutores en IE para asistir a la institución o como qué programas educativos que se fundamentan en el uso de las tecnologías de la información y la comunicación tienen un impacto en la visión de futuro.
Los coeficientes de correlación que arrojan valores inferiores a 0.30 generalmente deben ser desechados o reformulados, no solo porque producen una disminución de la confiablidad total de la escala, sino porque correlaciones a partir de 0.30 en general son estadísticamente significativas más allá del nivel del $1 \%$ (Cohen y Manion, 1990).

La otra explicación que existe para desechar los ítemes con coeficientes de correlación inferiores a 0.30 (debido a que se puede esperar diferencias significativas en coeficientes inferiores a ese valor), es que con el coeficiente correlación ( $r$ ) se calcula el Coeficiente de Determinación $(\pi)$ que explica el porcentaje de la variabilidad de un ítem con respecto del otro.

Con coeficientes de correlación inferiores a 0.3 , los coeficientes de determinación serán inferiores o iguales al 9\%, con lo que no se estaría explicando ni siquiera el $10 \%$ de la variabilidad de un ítem en comparación con el otro.

Una baja correlación entre los ítemes comparados y el puntaje total puede deberse a diversas causas, ya sea de mala redacción del ítem o que no funciona para medir lo que se desea, de ahí que la depuración de los ítemes tiene como consecuencia aumentar la fiabilidad de la escala.

Finalmente, la tercera prueba consiste en realizar un análisis de fiabilidad que permite estudiar las propiedades de las escalas de medición y de los ítemes que las constituyen.

La medida de la confiabilidad puede estimarse a través del coeficiente Alfa de Cronbach. La ventaja de este coeficiente reside en que requiere una sola administración del instrumento de medición y puede tomar valores entre 0 y 1 , donde 0 significa nula confiabilidad y 1 representa la confiabilidad total. La teoría ha venido considerando que un Alfa de Cronbach igual o superior a 0.7 
tiene una buena confiabilidad interna, tanto mejor cuanto más se acerque a 1 (Cohen y Manion, 1990).

El procedimiento permite calcular el Alfa de Cronbach para toda la escala y presenta el valor del coeficiente si el ítem es desechado. lo que ayuda a visualizar nuevos ítemes que no habían sido retirados con base en las dos pruebas anteriores, con el objetivo de aumentar la confiablidad de la escala.

Como se verá más adelante, estos tres procedimientos se aplicaron varias veces hasta llegar a la conformación de la EATLD final.

\section{Depuración de la escala inicial}

El objetivo de aplicar la escala a un grupo de tutores y maestros (as) de IE. antes de hacer la prueba para la metodología desarrollada, no es solo corroborar la redacción y el entendimiento de los ítemes sino tratar de reducir la cantidad de ítemes que contenía la escala.

Con respecto de la redacción y el entendimiento de los ítemes, se comprobó que ninguna de las afirmaciones producía problema. El análisis para determinar cuáles ítemes podrían excluirse de la escala se hizo con la aplicación de dos procesos.

El primero de ellos consistió en identificar ítemes que presentaran una variablidad nula (varianza cero), es decir aquellos para los cuales el coeficiente de correlación no se podía obtener debido a que las respuestas de maestros (as) y tutores (as) de IE eran constantes. Tres ítemes presentaron esta particularidad, todos pertenecientes a la categoría "Valoración del aprendizaje de la tecnología" aunque. debido a los objetivos del estudio y de la escala, dos de ellos se dejaron para administrar nuevamente la prueba a una mayor cantidad de personas. Estos fueron:

a. Vale la pena invertir en computadoras para el aprendizaje de las niñas (no fue excluido).

b. Que las niñas aprendan a utilizar las herramientas tecnológicas es muy importante (no fue excluido).

c. Que los (as) docentes aprendan a utilizar las herramientas tecnológicas es muy importante.

Posteriormente se obtuvo el coeficiente de correlación de todos los ítemes restantes y, con base en éste, se calculó el coeficiente de determinación para obtener la medida del porcentaje en que cada ítem explicaba la variabilidad de la categoría a la que pertenecía. Los ítemes que permanecieron en la escala fueron aquellos que explicaban al menos el $40 \%$ de la variablidad de la categoría. La escala resultó como sigue: 


\section{Valoración de la tecnología}

a. La tecnología ha beneficiado a la sociedad actual

b. Las herramientas tecnológicas mejoran la calidad de vida de sus usuarios (as)

c. El uso de la tecnología está cambiando positivamente la manera de estudiar de la gente

d. El uso de la tecnología está cambiando positivamente la manera de trabajar de las instituciones educativas

e. Las computadoras son muy caras para que las usen las niñas*

f. Las computadoras son muy caras para que las usen los niños*

2. Motivación para asistir a la institución

a. La mayoría de las veces tengo ganas de realizar las actividades que mi puesto me demanda

b. Creo que la tecnología no contribuye con que los (as) docentes se sientan más motivados (as) para asistir a su trabajo

c. Actualmente me siento motivado (a) para asistir a impartir lecciones

\section{Satisfacción con el programa o actividad}

a. La forma en que se implemente en mi institución el plan de estudios del MEP para primaria es satisfactoria para mí

b. Me siento satisfecho (a) en cuanto al aporte que hace el Programa al proceso de enseñanza - aprendizaje

c. Estoy satisfecho (a) en cuanto a las actividades que propone el Programa

d. En general, me siento satisfecho (a) respecto del funcionamiento del Programa en todos sus aspectos

e. Me siento satisfecho (a) en relación con el cumplimiento de los objetivos que propone el Programa 


\section{Tabla 1}

(Continuación)

\section{Conformación de la EATLD después de realizar la primera depuración}

\section{Autopercepción de la capacidad para enseñar}

a. Soy capaz de evaluar mi propio desempeño como docente

b. En general, pienso que mi desempeño profesional es adecuado

c. Pienso que mi profesión y mis conocimientos son insuficientes para la labor que desempeño en la escuela*

d. Me preocupo por mejorar constantemente mi desempeño profesional

e. Con respecto de la labor que desempeño en la escuela, pienso que mi formación y conocimientos son suficientes

f. En general, me siento satisfecho con mis capacidades para enseñar

g. En general, no me siento satisfecho con mis capacidades para enseñar*

h. Con respecto de la labor que desempeño como tutor (a) o maestro (a) de IE, pienso que mi formación y conocimientos son adecuados

i. Mi capacidad para enseñar se ha visto favorecida con las capacitaciones que he recibido en el Programa

j. Usar la tecnología en la escuela tiene un impacto positivo en la capacidad para enseñar de los y las docentes

$\mathrm{k}$. El uso de la tecnología ha tenido poca influencia en mi desempeño profesional

1. El uso de la tecnología me ha dado herramientas para mi desempeño profesional

m. Me siento satisfecho (a) en cuando a mi desempeño como tutor (a) o maestro (a) de IE

n. El uso de la tecnología ha tenido una influencia positiva en mi desempeño profesional

o. Mi desempeño como tutor (a) o maestro (a) de IE es inadecuado*

p. En general, me siento insatisfecho (a) en mi desempeño como tutor (a) o maestro (a) de IE

q. En general, pienso que mi desempeño como tutor (a) o maestro (a) de IE es adecuado 


\section{Visión de futuro}

a. Me interesa continuar estudiando formalmente

b. Tengo planes concretos para mejorar mi categoría profesional

c. En mi puesto actual tengo oportunidades para seguir enriqueciendo mi profesión

d. Aún tengo muchas cosas que aprender en mi profesión

e. Creo que dentro de 10 años mi situación económica será mucho mejor

f. Me interesa estar al tanto de los últimos avances de mi profesión

g. El uso de la tecnología contribuye con mi formación

h. El uso de la tecnología me ofrece oportunidades para mis planes futuros

i. El uso de la tecnología me ayuda a estar actualizado (a) en mi profesión

j. El uso de la tecnología contribuye con mi desarrollo académico

6. Sentido de control sobre la tecnología

a. Me siento nervioso (a) cuando trabajo con una computadora*

b. Usar las computadoras me frustra*

c. Las computadoras son difíciles de usar*

d. Un examen de computación me atemorizaría*

e. Me siento a gusto trabajando con una computadora

f. Puedo controlar tanto los recursos como las limitaciones que me ofrece la tecnología

g. Siento que las computadoras son más inteligentes que yo*

h. Creo que soy capaz de usar eficientemente una computadora

i. Creo que soy capaz de sacar provecho de las computadoras para mis labores docentes 


\section{Tabla 1}

\section{(Continuación)}

Conformación de la EATLD después de realizar la primera depuración

\section{Valoración del aprendizaje de la tecnología}

a. Vale al pena invertir en computadoras para el aprendizaje de las niñas

b. Creo que una política para dotar de herramientas tecnológicas a los centros educativos es muy importante

c. Que las niñas aprendan a usar las herramientas tecnológicas es muy importante

d. Vale la pena invertir en computadoras para el aprendizaje de los niños

e. El uso de las herramientas tecnológicas mejora el rendimiento de los y las estudiantes

f. Aprender a usar las herramientas tecnológicas es muy importante

g. Que los niños aprendan a usar las herramientas tecnológicas es muy importante

h. Incluir el aprendizaje de las herramientas tecnológicas en los plancs de estudio es muy importante

\section{Valoración de la acción pedagógica}

a. Generalmente mis estrategias pedagógicas me dan los resultados esperados en los y las estudiantes

b. A menudo no logro los resultados esperados en los y las estudiantes*

c. Tengo facilidad para darme a entender con los y las estudiantes*

\section{Motivación para aprender}

a. Estoy dispuesto (a) a adquirir nuevos conocimientos todos los días

b. Con los conocimientos que poseo me basta para desempeñarme adecuadamente en mi profesión*

c. Creo que ya aprendí todo lo que tenía que aprender* 


\section{Actitud para aprender nuevas habilidades relacionadas con la tecnología}
a. No me interesa aprender a utilizar las herramientas tecnológicas*
b. Estoy dispuesto (a) a aprender nuevas habilidades que se requieren para el uso de las herramientas tecnológicas
c. Quiero estar actualizado en cuanto al uso de las herramientas tecnológicas

\section{Actitud para gestionar medios externos}

a. Estoy dispuesto (a) a gestionar contactos entre la escuela y otras instituciones que la puedan ayudar

b. Pienso que es importante que la escuela tenga contacto con otras instituciones que la puedan ayudar

c. Es importante buscar recursos fuera de la institución educativa que permitan su desarrollo y mejor funcionamiento

* Ítem con valoración negativa

\section{Aplicación de la escala depurada}

La escala, después de la primera depuración, quedó conformada por 70 ítemes distribuidos en las mismas once categorías.

Para validarla en su segunda versión se hizo necesario aplicarla como mínimo a una cantidad de individuos igual a cinco veces el número de ítemes con el objetivo de evitar obtener correlaciones espuriamente altas, que pueden aparecer cuando el número de ítemes y el de individuos que responde la prueba son semejantes. La cantidad estimada de personas a las que debía administrarse el instrumento tenía que ser de 350.

Como ya se ha mencionado, la escala se aplicó a 319 maestros (as) y tutores (as) de IE (91\% del total sugerido) asistentes al IX Congreso Nacional Infantil de Informática Educativa, que se desarrolló en Santa Cruz, Guanacaste, los días 28 y 29 de marzo del 2003.

\section{Análisis de la segunda aplicación}

\section{a. Primer análisis}

Para iniciar con la depuración de la segunda aplicación de la escala, se sometió a un análisis de factores de componentes principales con rotación 
VARIMAX en dos momentos diferentes. En el primero se obtuvo el Diagrama de Sedimentación para determinar si las categorías construidas de previo agrupaban la mayor cantidad de ítemes posible, de manera que el análisis posterior permitiera limitar a 11 el número de categorías (o factores) involucradas.

Este análisis preliminar permitió comprobar que varios de los ítemes tenían cargas factoriales en categorías a las que no pertenecían originalmente. Este hecho indicaba que muy probablemente el conjunto de categorías no podría tomarse en su totalidad para realizar el análisis de factores, sino que era necesario dividirlas en subgrupos para analizar las cargas factoriales, según su lógica de medición y de pertenencia a una determinada temática. Si bien es cierto todos los ítemes están relacionados con la tecnología, se pueden identificar dos grandes grupos de categorías o dimensiones:

\section{Dimensión 1: Tecnología y su aprendizaje (26 ítemes)}

i. Valoración de la tecnología

ii. Sentido de control sobre la tecnología

iii. Valoración del aprendizaje de la tecnología

iv. Actitud para aprender nuevas habilidades relacionadas con la tecnología

Dimensión 2: Labor docente (44 ítemes)

i. Motivación para asistir a la institución

ii. Satisfacción con el programa o actividad

iii. Autopercepción de la capacidad para enseñar

iv. Visión de futuro

v. Valoración de la acción pedagógica

vi. Motivación para aprender

vii. Actitud para gestionar medios externos

En la Dimensión 1 el 96\% de los ítemes (25) registraron cargas factoriales iguales o mayores a 0.4 y en la Dimensión 2 el $91 \%$ de los ítemes (40) presentaron la misma condición. Posteriormente se obtuvieron los valores para los coeficientes de correlación de los ítemes en sus categorías de pertenencia y todos fueron mayores o iguales a 0.3 .

Si bien es cierto estos resultados garantizan una buena confiabilidad y consistencia interna de la EATLD, se hizo necesario realizar otros análisis para tratar de reducir la cantidad de ítemes de la escala. 


\section{b. Segundo análisis}

Una vez que fueron extraídos de la escala los ítemes que tenían una carga factorial menor a 0.4 con el procedimiento anterior, el segundo análisis se concentró en el cálculo de los coeficientes de correlación de los ítemes en las dimensiones respectivas, indistintamente de la categoría a la que pertenecían. Este procedimiento permite discriminar cuáles ítemes deberían de ser retirados de la escala para aumentar su confiabilidad, tomando como punto de referencia un valor mínimo obtenido en el coeficiente de correlación.

Debido a la necesidad de disminuir la cantidad de ítemes de la escala, se seleccionaron tres posibles valores del coeficiente de correlación, a través del cálculo del coeficiente de determinación, para obtener el porcentaje mínimo en que el ítem explica la variabilidad de la dimensión. Los valores utilizados fueron:

\section{Tabla 2}

Porcentaje mínimo explicado por el coeficiente de determinación según valores mínimos seleccionados para el coeficiente de correlación

\begin{tabular}{|c|c|c|}
\hline $\begin{array}{l}\text { Coeficiente de } \\
\text { Correlación (r) }\end{array}$ & $\begin{array}{c}\text { Coeficiente de } \\
\text { Determinación }(P)\end{array}$ & Interpretación \\
\hline$r \geq 0.30$ & $P \geq 10 \%$ & $\begin{array}{l}\text { El ítem explica al menos el } 10 \% \\
\text { de la variabilidad de la dimensión }\end{array}$ \\
\hline$r \geq 0.40$ & $P \geq 15 \%$ & $\begin{array}{l}\text { El ítem explica al menos el } 15 \% \\
\text { de la variabilidad de la dimensión }\end{array}$ \\
\hline$r \geq 0.45$ & $P \geq 20 \%$ & $\begin{array}{l}\text { El ítem explica al menos el } 20 \% \\
\text { de la variabilidad de la dimensión }\end{array}$ \\
\hline
\end{tabular}

Este procedimiento consiste en seleccionar ítemes dependiendo de un valor mínimo que se le haya asignado al coeficiente de correlación. En el Cuadro 1 se muestra la cantidad de ítemes seleccionados, dependiendo del valor de este coeficiente, para cada una de las dimensiones. 


\section{Cuadro 1}

\section{Cantidad de ítemes seleccionados según valores mínimos para el coeficiente de correlación}

\begin{tabular}{cccc}
\hline $\begin{array}{l}\text { Valor mínimo } \\
\text { del Coeficiente } \\
\text { de Correlación }\end{array}$ & $\begin{array}{c}\text { Cantidad de } \\
\text { ítemes en la } \\
\text { Dimensión 1 }\end{array}$ & $\begin{array}{c}\text { Cantidad de } \\
\text { ítemes en la } \\
\text { Dimensión 2 }\end{array}$ & $\begin{array}{c}\text { Total de } \\
\text { ítemes en la } \\
\text { EATLD }\end{array}$ \\
\hline$r \geq 0.30$ & 24 & 32 & 56 \\
$r \geq 0.40$ & 21 & 22 & 43 \\
$r \geq 0.45$ & 17 & 13 & 30 \\
\hline
\end{tabular}

Se observa, como es de esperar, que la cantidad de ítemes seleccionados disminuye conforme aumenta el límite inferior permitido en el coeficiente de correlación. En el primero ( $r \geq 0.30)$ se sigue teniendo una cantidad considerable de ítemes y la escala se ve reducida solo en un $14 \%$ de estos. En el caso de $r \geq 0.45$ se experimenta una reducción considerable de las afirmaciones de la escala (54\%) aunque, según el procedimiento, habría que desechar una gran cantidad de ítemes con valoraciones negativas que son de suma importancia dentro de la escala debido a que permiten establecer mecanismos de control en las respuestas (en comparación con las afirmaciones con valoración positiva) y tratan de eliminar la disposición de las personas entrevistadas a responder siempre de una misma forma.

Para el valor intermedio ( $r \geq 0.40$ ) se tiene una reducción del $34 \%$ de los ítemes en la EATLD y se mantiene una cantidad considerable de afirmaciones con valoración negativa. Debido a estas dos condiciones se tomó como punto de partida este valor del coeficiente de correlación para realizar el análisis final con base en los 43 ítemes resultantes.

Por lo tanto, el primer análisis permitió determinar que era necesario dividir las categorías en dos dimensiones y, a través del cálculo de las cargas factoriales y de los coeficientes de correlación, concluir que era necesario aplicar el procedimiento a distintos valores mínimos de estos últimos para lograr una reducción de ítemes en la escala, cuyos resultados fueron presentados en el segundo análisis. Una vez que fueron desechados de la escala todos los ítemes cuyos coeficientes de correlación fueron inferiores a 0.40 , se procedió nuevamente a calcular sus cargas factoriales, los nuevos coeficientes de correlación (volviendo a $\mathrm{r} \geq 0.30$ ) y los coeficientes de confiabilidad (Alfa de Cronbach). Este análisis se presenta a continuación. 


\section{c. Tercer análisis}

Con este tercer análisis se buscó una reducción final de los ítemes de la escala. tomando como punto de partida la cantidad de ítemes resultantes de los dos anteriores (43). El procedimiento se presenta para cada una de las dimensiones de la EATLD.

\section{Dimensión 1}

Para este primer grupo el Diagrama de Sedimentación de los ítemes corroboró que la mayoría de estos podían ubicarse en cuatro categorías, aunque los resultados arrojaron que algunos deberían estar colocados en otras categorías y no necesariamente en las de partida. La selección final de los ítemes por categoría que puntuaron como mínimo $\mathbf{0}$.4 se muestran en el siguiente cuadro.

\section{Cuadro 2}

\section{Cargas factoriales de los ítemes de la dimensión "Tecnología y su aprendizaje"}

\begin{tabular}{lcccc}
\hline & \multicolumn{3}{c}{ Factores } \\
\cline { 2 - 4 } & $\mathbf{1}$ & $\mathbf{2}$ & $\mathbf{3}$ & $\mathbf{4}$ \\
\hline Valor propio & 3.42 & 3.33 & 2.18 & 1.71 \\
Varianza explicada & $16.3 \%$ & $15.9 \%$ & $10.4 \%$ & $8.2 \%$ \\
\hline
\end{tabular}

\section{İtemes}

Las computadoras son muy caras para que las usen los niños $\quad 0.85$

Las computadoras son muy caras para que las usen las niñas $\quad 0.77$

Creo que soy capaz de usar eficientemente una computadora $\quad 0.80$

$\begin{array}{ll}\text { Siento que las computadoras son más inteligentes que yo } & 0.70\end{array}$

Creo que soy capaz de sacar provecho de las computadoras para mis labores docentes

Puedo controlar tanto los recursos como las limitaciones que me ofrece la tecnología

0.68

Quiero estar actualizado (a) en cuanto al uso de las herramientas tecnológicas

Aprender a usar las herramientas tecnológicas es muy importante

Que las niñas aprendan a usar las herramientas tecnológicas es muy importante

Que los niños aprendan a utilizar las herramientas tecnológicas es muy importante

Vale la pena invertir en computadoras para el aprendizaje de las niñas

Vale la pena invertir en computadoras para el aprendizaje de los niños 
Cuadro 2

(Continuación)

\section{Cargas factoriales de los ítemes de la dimensión "Tecnología y su aprendizaje"}

\begin{tabular}{|c|c|c|c|c|}
\hline & \multicolumn{4}{|c|}{ Factores } \\
\hline & 1 & 2 & 3 & 4 \\
\hline $\begin{array}{l}\text { Vale la pena invertir en computadoras para el aprendizaje de } \\
\text { los niños }\end{array}$ & 0.56 & 0.43 & & \\
\hline $\begin{array}{l}\text { Estoy dispuesto (a) a aprender nuevas habilidades que se } \\
\text { requieren para el uso de las herramientas tecnológicas }\end{array}$ & & 0.48 & & \\
\hline No me interesa aprender a usar las herramientas tecnológicas & 0.41 & 0.48 & & \\
\hline $\begin{array}{l}\text { El uso de las herramientas tecnológicas mejora el rendimiento } \\
\text { académico de los y las estudiantes }\end{array}$ & & 0.45 & & \\
\hline Me siento a gusto trabajando con una computadora & & & 0.41 & \\
\hline Me siento nervioso cuando trabajo con una computadora & & & & 0.70 \\
\hline Usar las computadoras me frustra & & 0.44 & & 0.59 \\
\hline Un examen de computación me atemorizaría & & & & 0.56 \\
\hline Las computadoras son difíciles de usar & & & & 0.49 \\
\hline
\end{tabular}

Nota: Solo se presentan los ítemes que tuvieron una carga factorial mayor o igual a 0.4. Además, puede observarse que algunos ítemes presentan cargas en más de un factor: para efectos del análisis se tomará la pertenencia del ítem a la categoría donde la carga factorial . registra un mayor valor.

Según los cálculos obtenidos el primer factor, con un valor propio de 3.42, explica el $16.3 \%$ de la varianza de los ítemes y por su tipo se le ha denominado "Valoración de la tecnología". El segundo factor explica el $15.9 \%$ de la varianza de las respuestas y tiene un valor propio de 3.73 y, debido a la mayoría de las afirmaciones que lo conforman se le ha dado el nombre de "Valoración del aprendizaje de la tecnología". El tercer factor tiene un valor propio de 2.18, explica el $10.4 \%$ de la variablidad de sus ítemes y, debido a esta conformación, ha sido llamado "Autopercepción del control sobre la tecnología". Finalmente, el cuarto factor explica el $8.2 \%$ de la variabilidad y posee un valor propio de 1.71; debido a las frases que lo conforman se ha nombrado como "Disposición hacia el uso de la tecnología".

\section{Dimensión 2}

El Diagrama de Sedimentación para este grupo mostró una clara agrupación de la mayoría de los ítemes alrededor de tres categorías. La selección de los ítemes con carga factorial mayor 0.4 se describen en el Cuadro 3. 
Como se puede observar nueve de los ítemes han quedado concentrados alrededor del factor 1 cuyo nombre es "Valoración de la tecnología en relación con el desarrollo profesional"; este factor tiene un valor propio de 3.81 y explica el 17.3\% de la variabilidad de sus ítemes. El factor 2 tiene un $14.5 \%$ de varianza explicada y posee un valor propio de 3.20, agrupando ítemes en la nueva categoría denominada "Satisfacción con el funcionamiento del Programa". La categoría "Autopercepción del desempeño" (factor 3) tiene un valor propio de 3.15 y explica el $14.3 \%$ de la varianza de las respuestas.

El nuevo análisis de factores permitió desechar un ítem en cada una de las dimensiones (con cargas factoriales inferiores a 0.4) obteniéndose, hasta este momento, un total de 41.

Una de las ventajas de reacomodar los ítemes en las categorías como consecuencia del análisis de factores es que se obtiene una buena correlación ítemtotal. Los Cuadros 4 y 5 muestran los valores de los coeficientes de correlación para cada uno de los ítemes con respecto a sus categorías de pertenencia en cada una de las dos dimensiones.

Todas las correlaciones de los ítemes con sus categorías respectivas fueron mayores a 0.30 , con lo que se garantiza que al menos un $10 \%$ de la variabilidad de las categoría se explica por los ítemes que las componen. Los resultados de las correlaciones fueron significativas al $1 \%$.

\section{Cuadro 3}

Cargas factoriales de los ítemes de la dimensión "Labor docente"

\begin{tabular}{|c|c|c|c|}
\hline & \multicolumn{3}{|c|}{ Factores } \\
\hline & 1 & 2 & 3 \\
\hline Valor propio & 3.42 & 3.33 & 2.18 \\
\hline Varianza explicada & $16.3 \%$ & $15.9 \%$ & $10.4 \%$ \\
\hline \multicolumn{4}{|l|}{ Ítemes } \\
\hline El uso de la tecnología contribuye con mi desarrollo académico & 0.66 & 0.45 & \\
\hline Con respecto de la labor que desempeño como tutor o maestro de & & & \\
\hline IE, pienso que mi profesión y mis conocimientos son suficientes & & 0.62 & \\
\hline Mi desempeño como tutor o maestro de IE es inadecuado & & 0.61 & \\
\hline $\begin{array}{l}\text { El uso de la tecnología ha tenido poca influencia en mi desempeño } \\
\text { profesional }\end{array}$ & 0.64 & & \\
\hline $\begin{array}{l}\text { El uso de la tecnología me ha dado herramientas para mi desempeño } \\
\text { profesional } \\
\text { El uso de la tecnología me ayuda a estar actualizado en mi profesión }\end{array}$ & 0.60 & 0.52 & \\
\hline \multirow{2}{*}{$\begin{array}{l}\text { El uso de la tecnología ha tenido una influencia positiva en mi } \\
\text { desempeño profesional }\end{array}$} & 0.60 & 0.52 & \\
\hline & 0.60 & 0.49 & \\
\hline
\end{tabular}




\section{Cuadro 3}

(Continuación)

Cargas factoriales de los ítemes de la dimensión "Labor docente"

\begin{tabular}{lccc}
\hline & \multicolumn{3}{c}{ Factores } \\
\cline { 2 - 4 } & $\mathbf{1}$ & $\mathbf{2}$ & $\mathbf{3}$ \\
\hline Valor propio & 3.42 & 3.33 & 2.18 \\
Varianza explicada & $16.3 \%$ & $15.9 \%$ & $10.4 \%$ \\
\hline
\end{tabular}

\section{Ítemes}

El uso de la tecnología me ofrece oportunidades para mis planes $\quad \begin{array}{lll}0.55 & 0.52\end{array}$ fururos

El uso de la tecnología contribuye con mi formación

Tengo planes concretos para mejorar mi categoría profesional

En general, pienso que mi desempeño profesional es adecuado

Usar la tecnología en la escuela tiene un impacto positivo en la 0.46 calidad para enseñar de los y las docentes

Me preocupo constantemente por mejorar mi desempeño profesional

Creo que dentro de 10 años mi situación socioeconómica será mucho mejor

Estoy satisfecho en cuanto a las actividades que propone el Programa

En general, me siento satisfecho respecto del funcionamiento del Programa en todos sus aspectos

Me siento satisfecho (a) en relación con el cumplimiento de los objetivos que propone el Programa

Me siento satisfecho (a) en cuanto al aporte que hace el Programa al proceso de enseñanza - aprendizaje

La mayoría de las veces tengo ganas de realizar las actividades que mi puesto demanda

Actualmente me siento motivado (a) para asistir a impartir lecciones

Nota: Solo se presentan los ítemes que tuvieron una carga factorial mayor o igual a 0.4. Además, puede observarse que algunos ítemes presentan cargas en más de un factor; para efectos del análisis se tomará la pertenencia del ítem a la categoríadonde la carga factorial registra un mayor valor 


\section{Cuadro 4}

\section{Coeficientes de correlación de los ítemes de la dimensión "Tecnología y su aprendizaje"}

\begin{tabular}{|c|c|c|c|c|}
\hline \multirow{2}{*}{ Ítemes } & \multicolumn{4}{|c|}{ Categorías $^{1}$} \\
\hline & 1 & 2 & 3 & 4 \\
\hline $\begin{array}{l}\text { Las computadoras son muy caras para que las usen los } \\
\text { niños }\end{array}$ & $0.87 * *$ & & & \\
\hline Las computadoras son muy caras para que las usen las niñas & $0.88 * *$ & & & \\
\hline $\begin{array}{l}\text { Vale la pena invertir en computadoras para el aprendizaje de } \\
\text { las niñas }\end{array}$ & $0.51 * *$ & & & \\
\hline $\begin{array}{l}\text { Vale la pena invertir en computadoras para el aprendizaje de } \\
\text { los niños }\end{array}$ & $0.63 * *$ & & & \\
\hline
\end{tabular}

Quiero estar actualizado (a) en cuanto al uso de las

herramientas tecnológicas

Aprender a usar las herramientas tecnológicas es muy

importante

Que las niñas aprendan a usar las herramientas tecnológicas

es muy importante

Que los niños aprendan a usar las herramientas tecnológicas

es muy importante

No me interesa aprender a utilizar las herramientas

tecnológicas

$0.63 * *$

Estoy dispuesto (a) a aprender nuevas habilidades que se

requieren para el uso de la tecnología

$0.69 * *$

El uso de las herramientas tecnológicas mejora el

rendimiento de los y las estudiantes

$0.67 * *$

Creo que soy capaz de usar eficientemente una

computadora

Creo que soy capaz de sacar provecho de las computadoras

para mis labores docentes

$0.74 * *$

Siento que las computadoras son más inteligentes que yo

$0.61 * *$

Puedo controlar tanto los recursos como las limitaciones

que me ofrece la tecnología

$0.56 * *$

Me siento a gusto trabajando con una computadora

$0.59 * *$

Me siento nervioso (a) cuando trabajo con una computadora

Usar las computadoras me frustra

Un examen de computación me atemorizaría

Las computadoras son difíciles de usar

** $\quad \mathrm{p}<0.01$

${ }^{1}$ Las categorías son:

a. Valoración de la tecnología

b. Valoración del aprendizaje de la tecnología

c. Autopercepción del control sobre la tecnología

d. Disposición hacia el uso de la tecnología 
La categoría "Disposición hacia el uso de la tecnología" está conformada solo por ítemes negativos. En este sentido, puede existir la posibilidad de que compartan variabilidad porque miden el mismo constructo o porque todos son negativos. Por lo tanto, a pesar de los valores altos obtenidos en la correlación, sería recomendable balancear esta categoría con ítemes positivos para tener la seguridad de que miden la disposición hacia el uso de la tecnología y no la posible tendencia de los sujetos a responder ítemes negativos de alguna forma específica. En aplicaciones posteriores de la escala final recomendada, podrían hacerse estas mediciones a una mayor cantidad de personas y volver a determinar las cargas factoriales para observar si los ítemes de esta categoría se agrupan con algunos otros que son positivos.

Por otro lado, es importante obtener la correlación de las categorías en relación con la dimensión a la que pertenecen, de manera que se pueda determinar el porcentaje de explicación de la variabilidad de las dimensiones con sus categorías. Este resumen se obtuvo sumando los puntajes obtenidos en cada uno de los ítemes para una categoría específica y dividiéndolo entre la totalidad de estos. En el Cuadro 6 se resume esta información.

\section{Cuadro 5}

\section{Coeficientes de correlación de los ítemes de la dimensión "Labor docente"}

\begin{tabular}{llc}
\hline Ítemes & \multicolumn{2}{c}{ Categorías $^{1}$} \\
\cline { 3 - 3 } & $\mathbf{1}$ & $\mathbf{2}$ \\
\hline El uso de la tecnología me ayuda a estar actualizado (a) en mi & & \\
profesión & $0.78^{* *}$ & \\
El uso de la tecnología me ofrece oportunidades para mis planes & & \\
futuros & $0.77^{* *}$ \\
El uso de la tecnología contribuye con mi formación & $0.76^{* *}$ \\
El uso de la tecnología contribuye con mi desarrollo académico & $0.79^{* *}$ \\
El uso de la tecnología ha tenido poca influencia en mi desempeño & \\
profesional & $0.58^{* *}$ \\
$\begin{array}{l}\text { El uso de la tecnología ha tenido una influencia positiva en mi } \\
\text { desempeño profesional }\end{array}$ & $0.74^{* *}$ \\
$\begin{array}{l}\text { El uso de la tecnología me ha dado herramientas para mi desempeño } \\
\text { profesional }\end{array}$ & $0.77^{* *}$ \\
$\begin{array}{l}\text { Usar la tecnología en la escuela tiene un impacto positivo en la } \\
\text { capacidad para enseñar de los y las docentes }\end{array}$ & $0.54 * *$ \\
\hline
\end{tabular}




\section{Coeficientes de correlación de los ítemes de la dimensión "Labor docente"}

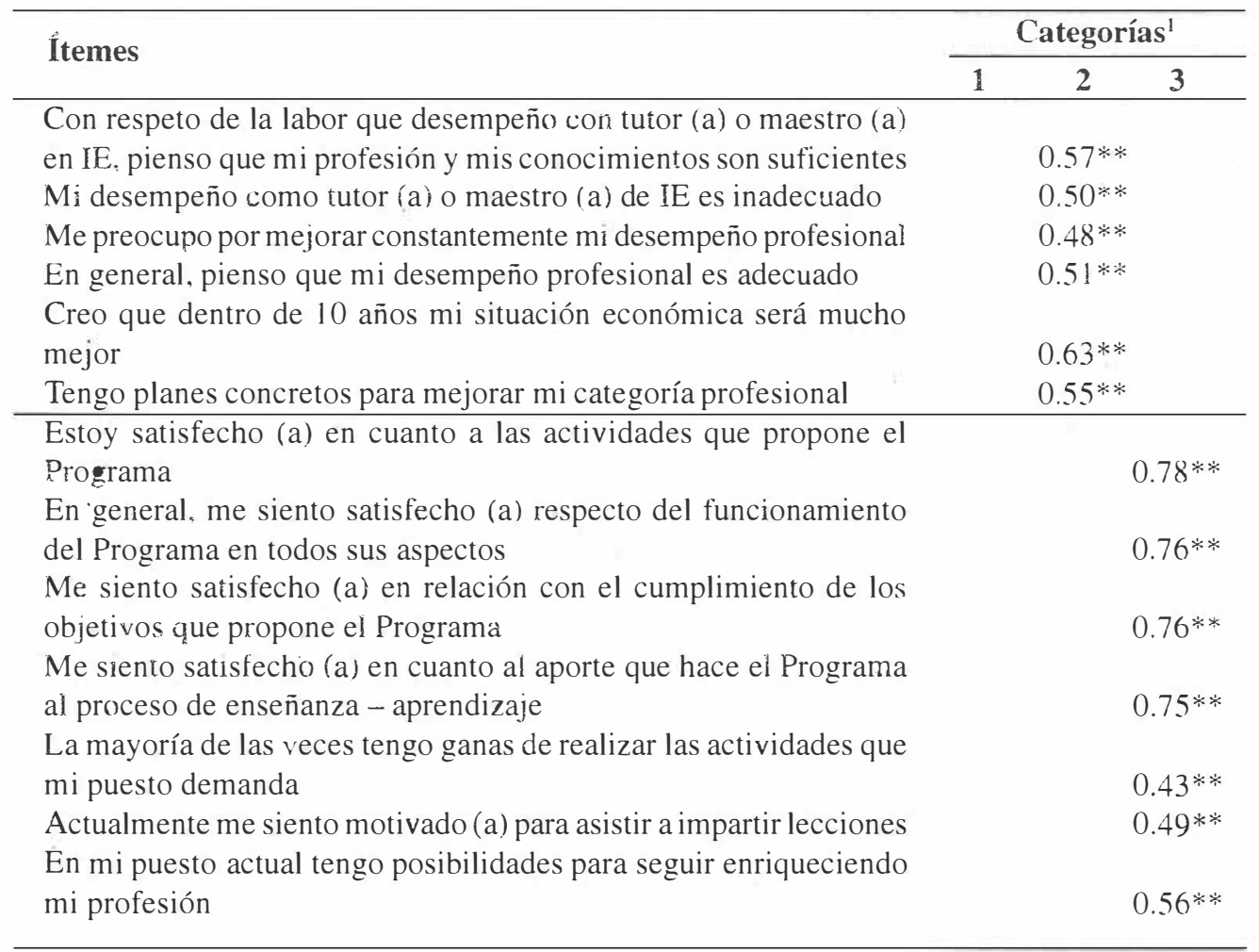

$* * \mathrm{p}<0.01$

'Las categorías son:

1. Valoración de la tecnología en relación con el desarrollo profesional

2. Autopercepción del desempeño

3. Satisfacción con el funcionamiento del Programa 


\section{Cuadro 6 \\ Coeficientes de correlación de las categorías según la dimensión a la que pertenecen}

\begin{tabular}{lcc}
\hline \multirow{2}{*}{ Categorías } & \multicolumn{2}{c}{ Dimensiones } \\
\cline { 2 - 2 } & $\mathbf{1}$ & $\mathbf{2}$ \\
\hline Valoración de la tecnología & $0.82 * *$ \\
Valoración del aprendizaje de la tecnología & $0.79 * *$ \\
Autopercepción del control sobre la tecnología & $0.80 * *$ \\
Disposición hacia el uso de la tecnología & $0.72 * *$ \\
\hline Valoración de la tecnología en relación con el desarrollo profesional & $0.83^{* *}$ \\
Autopercepción del desempeño & $0.79 * *$ \\
Satisfacción con el funcionamiento del Programa & $0.80^{* *}$ \\
\hline Escala total & $0.88^{* *}$ & $0.86^{* *}$ \\
\hline
\end{tabular}

$* * p<0.01$

Todas las correlaciones de las categorías con sus respectivas dimensiones fueron significativas al $1 \%$ y mayores o iguales a 0.3 . El procedimiento que se aplicó para obtener las correlaciones de las categorías con sus respectivas dimensiones también se empleó para calcular las correlaciones de las dimensiones con respecto de la escala total. En este aspecto también se obtuvieron correlaciones significativas al $1 \%$ y mayores a 0.3 (0.88 en la Dimensión 1 y 0.86 en la Dimensión 2). Finalmente, la correlación entre las dos dimensiones es de 0.53 (significativa al $1 \%$ ). Este valor garantiza que hay una buena correlación entre cada una de las dimensiones, por lo que se puede concluir que la EATLD debe estar conformada por las dos dimensiones para su aplicación. Con el análisis de los coeficientes de correlación se obtuvieron nuevamente 20 ítemes involucrados en la Dimensión 1 y 21 en la Dimensión 2, divididos en 4 y 3 categorías respectivamente.

Dado que el análisis de factores arrojó resultados en torno a dos dimensiones. para el análisis de fiabilidad se introdujeron los ítemes correspondientes a cada una de las dimensiones por separado. Por lo general, si en el análisis de confiabilidad se obtienen Alfas mayores o iguales a 0.7 y coeficientes de correlación mayores o iguales a 0.3 se puede concluir que hay una buena consistencia interna de la escala.

En el caso de la Dimensión 1 el Alfa de Cronbach fue de 0.8302 y en la Dimensión 2 de 0.8524 . Para la totalidad de la escala fue de 0.8985 , con lo que se puede concluir que existe una muy buena consistencia interna de la escala, tanto de los ítemes en sus categorías como de éstas en sus dimensiones.

Si bien es cierto se solicitó el cálculo de los Alfas si los ítemes eran borrados de la escala, tanto para las dimensiones individuales como para la escala total, el retiro de alguno no producía variaciones importantes (de incremento) en el Alfa de Cronbach. 


\section{La conformación de la escala final}

La siguiente es la propuesta final de la Escala de actitudes hacia la tecnología en la labor docente, una vez que se determinaron los ítemes que arrojaban una carga factorial superior o igual a 0.4 , los coeficientes de correlación fueron todos mayores o iguales a 0.3 (donde todas las correlaciones resultaron significativas al 1\%) y cuyo análisis de confiabilidad dio como resultado un Alfa de Cronbach de 0.8985 .

\section{Tabla 3}

\section{Escala de actitudes hacia la tecnología en la labor docente Vargas, Castro, Zúñiga y Rodríguez}

\section{Dimensión 1: Tecnología y su aprendizaje}

Categoría 1: Valoración de la tecnología

Las computadoras son muy caras para que las usen los niños* Las computadoras son muy caras para que las usen las niñas* Vale la pena invertir en computadoras para el aprendizaje de los niños

Vale la pena invertir en computadoras para el aprendizaje de las niñas

\section{Categoría 2: Valoración del aprendizaje de la tecnología}

Quiero estar actualizado (a) en cuanto al uso de las herramientas tecnológicas

Aprender a usar las herramientas tecnológicas es muy importante

Que las niñas aprendan a utilizar las herramientas tecnológicas es muy importante

Que los niños aprendan a utilizar las herramientas tecnológicas es muy importante No me interesa aprender a usar las herramientas tecnológicas*

Estoy dispuesto (a) a aprender nuevas habilidades que se requieren para el uso de las herramientas tecnológicas

El uso de las herramientas tecnológicas mejora el rendimiento académico de los y las estudiantes

\section{Categoría 3: Autopercepción del control sobre la tecnología}

Creo que soy capaz de usar eficientemente una computadora

Creo que soy capaz de sacar provecho de las computadoras para mis labores docentes Siento que las computadoras son más inteligentes que yo*

Puedo controlar tanto los recursos como las limitaciones que me ofrece la tecnología Me siento a gusto trabajando con una computadora

\section{Categoría 4: Disposición hacia el uso de la tecnología}

Me siento nervioso (a) cuando trabajo con una computadora*

Usar las computadoras me frustra*

Un examen de computación me atemorizaría*

Las computadoras son difíciles de usar* 


\section{Tabla 3}

(Continuación)

\section{Escala de actitudes hacia la tecnología en la labor docente Vargas, Castro, Zúñiga y Rodríguez}

\section{Dimensión 2: Labor docente}

Categoría 1: Valoración de la tecnología en relación con el desarrollo profesional

El uso de la tecnología me ayuda a estar actualizado (a) en mi profesión

El uso de la tecnología me ofrece oportunidades para mis planes futuros

El uso de la tecnología contribuye con mi formación

El uso de la tecnología contribuye con mi desarrollo académico

El uso de la tecnología ha tenido poca influencia en mi desempeño profesional*

El uso de la tecnología ha tenido una influencia positiva en mi desempeño profesional

El uso de la tecnología me ha dado herramientas para mi desempeño profesional

Usar la tecnología en la escuela tiene un impacto positivo en la capacidad para enseñar de los y las docentes

\section{Categoría 2: Satisfacción con el funcionamiento del Programa}

Estoy satisfecho (a) en cuanto a las actividades que propone el Programa

En general, me siento satisfecho (a) respecto del funcionamiento del Programa en todos sus aspectos

Me siento satisfecho (a) en relación con el cumplimiento de los objetivos que propone el Programa

Me siento satisfecho (a) en cuanto al aporte que hace el Programa al proceso de enseñanza - aprendizaje

La mayoría de las veces tengo ganas de realizar las actividades que mi puesto demanda Actualmente me siento motivado (a) para asistir a impartir lecciones

En mi puesto actual tengo posibilidades de seguir enriqueciendo mi profesión

\section{Categoría 3: Autopercepción del desempeño}

Con respecto de ìa labor que desempeño como tutor (a) o maestro (a) de inf •rmática educativa, pienso que mi profesión y mis conocimientos son suficientes

Mi desempeño como tutor (a) o maestro (a) de informática educativa es inadecuado* Me preocupo constantemente por mejorar mi desempeño profesional

En general, pienso que mi desempeño profesional es adecuado

Creo que dentro de 10 años mi situación económica será mucho mejor

Tengo planes concretos para mejorar mi categoría profesional

* Ítem con valoración negativa 


\section{Conclusiones}

La validez y la confiabilidad son dos conceptos de suma importancia en la aplicación de una escala. cualquiera que sea el tema.

Cuando se construye una escala conformada por categorías cuya definición fue previa a su aplicación, la validez y la confiabilidad permiten determinar lá homogeneidad de los constructos y cuán confiable puede ser la escala para medir lo que se desea medir.

En el caso de la Escala de actitudes hacia la tecnología en la labor docente, este análisis debió realizarse en tres partes, debido a que con el primero se determinó que con el cálculo de las cargas factoriales y de las correlaciones (ambos valores altos para casi la totalidad de afirmaciones) no era posible reducir significativamente la cantidad de ítemes en la escala.

Con el análisis de factores inicial se descubrió que era necesario desechar ítemes que no estaban aportando a la homogeneidad, reacomodar algunos que puntuaban más alto en relación con otros que pertenecían a categorías distintas y hacer dos grandes grupos de categorías que se relacionaran temáticamente y que aumentaran la confiabilidad de la escala.

Por otro lado, el reacomodo de los ítemes en otras categorías sugirió, a través del análisis de las diferentes afirmaciones que las conformaban, que era necesario renombrarlas para que sus ejes temáticos se vieran enriquecidos por el nuevo acomodo. Además, se garantizó que las correlaciones fueran altas y significativas.

Las pruebas relacionadas con el análisis de factores y el cálculo de los coeficientes de correlación confluyeron en la obtención de un Alfa de Cronbach de 0.8985 , lo que asegura una muy buena consistencia interna de la escala.

De 115 ítemes distribuidos en once categorías, se obtuvo al final una escala con 41 ítemes distribuidos en siete categorías y éstas en dos dimensiones.

Se ha presentado un análisis exhaustivo a nivel de confiabilidad. Sin embargo, es importante recordar que el análisis de factores es exploratorio, es decir, depende en gran parte de las respuestas de las personas y no solo de la teoría que sustenta la construcción de una escala, por lo que se podría concluir que el análisis de factores debe verse complementado con otros criterios para comprobar la validez, tales como los criterios de validez convergente (la escala correlaciona con otras que miden constructos parecidos), validez discriminante (la escala no correlaciona con otras que miden temas distintos) o validez predictiva (puede predecir la escala, por ejemplo, el desempeño de los (as) docentes). 


\section{Referencias}

Amón, J. (1993). Estadística para psicólogos 2. Madrid: Ediciones Pirámide.

Castro, R. (2003). Actitudes de tutores y tutoras de informática educativa hacia la tecnología en la educación y el aprendizaje. Documento de trabajo. Departamento de Investigación, Fundación Omar Dengo.

Cohen, L. y Manion, L. (1990). Métodos de investigación educativa. España: Ediciones La Muralla.

Downie, N. y Heath, R. (1973). Métodos estadísticos aplicados. México D.F.: HARLA, S.A.

Franton, C. Green, K. y Hoffman, E. (2002). Measure Development: The Children's Attitudes Toward Technology Scale (CATS). En: Journal of Educational Computing Research. 26(3), 249-263.

Hernández, R. et al. (2000). Metodología de la investigación. México D.F.: Mc Graw Hill.

Lignan, L. (2002). Actitudes de los alumnos y maestros hacia la computadora y losmedios para el aprendizaje. Publicación en línea http://investigacion.ilce.edu.mx dice/proyectos/actitudes/actit9.htm. Fecha de consulta: 9 de octubre del 2003.

Lignan, L. (2002). Validación del cuestionario sobre las actitudes de los maestros hacia la computadora. Documentos de la Sociedad Mexicana de Computación, México. Publicación en línea http://www.te.ipn.mx/files/f000504/ txt.docto.htm. Fecha de consulta: 27 de febrero del 2003.

Mitra, A. (2002). Toward Developing Questionnaire Items to Measure Effectiveness of Computers in Teaching. En: Journal of Educational Computing Research. 26(4), 381-394.

Myint Swe, K. (2001). Attitudes Toward Computers Among Teachers Education Students in Brunei Darussalam. En: International Journal of Instructional Media. 28(2), 147. 
Rodríguez, J. (2002). Marco evaluativo de impacto social con perspectiva de equidad para programas educativos mediados por TIC. Documento de trabajo. Departamento de Investigación, Fundación Omar Dengo.

Rodríguez. J. (2002). La investigación evaluativa de programas educativos con TIC: propuesta del estado del arte. Departamento de Investigación. Fundación Omar Dengo.

Steel, R. y Torri, J. (1986). Bioestadística. Principios y procedimientos. México D.F.: Mc Graw Hill. 